\title{
ESTIMATIVA DA ERODIBILIDADE A PARTIR DE ATRIBUTOS DE SOLOS COM HORIZONTE B TEXTURAL NO BRASIL ${ }^{(1)}$
}

\author{
J J .G.S.M. MARQUES ${ }^{(2)}$, N. CURI ${ }^{(3)}$, J .M. de LIMA ${ }^{(3)}$, M.M. FERREIRA ${ }^{(3)}$, \\ M.L.N. SILVA ${ }^{(4)} \&$ D.F. FERREIRA ${ }^{(5)}$
}

\begin{abstract}
RESUMO
Os métodos existentes para estimar a erodi bilidade dos solos (fator K) não se têm mostrado adequados quando aplicados a solos brasileiros. Este trabalho teve por objetivo o desenvolvimento de método indireto para estimar a erodibilidade, visando obter esse fator, a partir de propriedades do solo facilmente determináveis. Assim, os horizontes A e Bt (B textural) de 22 solos de várias regióes geográficas do Brasil foram submetidos a diversas análises químicas e físicas, e a descrição morfológica do perfil foi considerada. Tais resultados (variáveis independentes) foram submetidos à análise de regressão linear múltipla em "stepwise", e relacionados com a erodibilidade (fator K) medida diretamente no campo (variável dependente). Obtiveram-se várias equações com graus crescentes de complexidade e precisão. A partir das variáveis selecionadas, tanto do horizonte A quanto do Bt, e da distribuição do tamanho das partículas, obti da com e sem dispersante químico, foi possível estimar, satisfatoriamente, a erodi bilidade desses solos. As informações contidas na descrição morfológica dos perfis de solo, especialmente as referentes à estrutura, bem como os teores de carbono orgânico, foram de grande importância para a determi nação indireta da erodibilidade (fator K).
\end{abstract}

Termos de indexação: erosão, erodi bilidade do solo (fator K), equação universal de perdas de solo.

\section{SUMMARY: ESTIMATION OF ERODIBILITY FROM ATTRIBUTES OF SOILS WITH ARGILLIC HORIZON IN BRAZIL}

The soil erodibility (K-factor) determination by indirect methods has not been adequate for application in Brazilian soils. This paper aimed the development of K-factor evaluation methods depending on easily-assessed chemical, physical and morphological soil properties. Samples from A and B horizons of 22 Brazilian soils bearing argillichorizon weresubmitted to several chemical and physical analyses and the morphological description of the soil profile

\footnotetext{
(1) Parte da dissertação de Mestrado apresentada pel o primeiro autor à U niversidade Federal de Lavras (MG). Recebido para publicação em julho de 1996 e aprovado em agosto de 1997.

(2) Aluno de Mestrado do CPGSNP- Universidade Federal de Lavras, Caixa Postal 37, CEP 37200-000 Lavras (MG).

(3) Professor, UFLA - Departamento de Ciência do Solo, Caixa Postal 37, CEP 37200-000 Lavras (MG). Bolsista do CNPq.

(4) Pesquisador, E MBRAPA - Centro Nacional de Pesquisa de Solos, Rua J ardim Botânico, 1024, CEP 22460-000 Rio de J aneiro (RJ ).

(5) Professor, UFLA - Departamento de Ciências Exatas, Caixa Postal 37, CEP 37200-000 Lavras (MG).
} 


\begin{abstract}
was considered. A stepwise multi ple linear regression analysis was run, relating the results of those analyses with the K-factor values directly measured in the field. Several equations with increasing degrees of complexity and precision were obtained. From the sel ected variables from the $A$ and $B$ horizons, as well as the particlesize distribution with and without chemical dispersant, it was possibleto satisfactorily estimatetheerodibility of such soils. Theinformations obtained from the soil profile description, specially about soil structure, as well as organic carbon amounts, are of major importance for the indirect determination of the erodibility (Kfactor).
\end{abstract}

Index terms: erosion, soil erodibility (K-factor), Universal Soil Loss Equation (USLE).

\section{INTRODUÇÃO}

A demanda por modelos que permitam estimar a er osão hídrica causada pel o uso do solo écrescente. A equação universal de perdas de solo - USLE (Wischmeier \& Smith, 1978), apesar de suas restrições (Wischmeier, 1976), é considerada o mais eficiente modelo de estimativa de perdas de solo, sendo amplamente utilizada, principalmente nos EUA (Renard et al., 1994). Embora os princípios da USLE possam ser aplicados às mais diversas situações, daí o fato de ser chamada de "universal", ela só pode ser usada com sucesso em situações em que os parâmetros envolvidos sejam determinados local mente (Foster et al., 1982).

A erodibilidade do solo, representada na USLE pelo fator $\mathrm{K}$, é, indubitavel mente, mais bem determinada por meio da instalação de parcelas de perdas de solo no campo. Entretanto, uma vez que a instalação e a manutenção dessas parcelas envolvem custos consideráveis, além de demandar vários anos de coleta de dados, muitas vezes opta-se por determinar o fator $\mathrm{K}$ por meio de chuvas simuladas. Embora isso apresente a vantagem da maior rapidez, a simulação de chuvas não é ainda prática o bastante para ser usada em grande número de solos em vários locais (Wischmeier \& Mannering, 1969). Por esses motivos, foram desenvolvidas equações, relacionando o fator $\mathrm{K}$, medido diretamente no campo, com atributos do sol o relativos à resistência do sol o à erosão, com vistas em estimar a erodibilidade para sol os onde o referido fator não é medido diretamente (Wischmeier et al., 1971; Römkens et al., 1977; Young \& Mutchler, 1977; Denardin, 1990).

Dada a variação entre solos, o fator $\mathrm{K}$ é o mais difícil de ser adaptado a condições diferentes daquelas usadas na el aboração dos model os (Foster et al ., 1982). Por esta razão, os métodos indiretos para estimativa desse fator, desenvolvidos para solos de regiões temperadas, não se têm mostrado adequados aos solos de regiões tropicais (Henklain \& Freire, 1983; Silva et al., 1986; Obi et al., 1989; Denardin, 1990; Lima, 1991; Silva et al., 1994; Marques, 1996). Mesmo métodos desenvol vidos, ou adaptados, para oambiente tropical, como aqueles apresentados por Denardin (1990) e Lima et al. (1990), não se têm mostrado satisfatórios (Silva et al., 1994; Marques, 1996). Possivel mente, uma das causas dessa inadequação é devido à grande heterogeneidade do comportamento dos solos tropicais frente aos processos erosivos (EI-Swaify \& Dangler, 1982). Por esse motivo, propôsse desenvolver equações de estimativa do fator $K$ específicas a cada grupamento de solos, ao invés de um único método para todo o universo de solos existentes em dada região (EI-Swaify \& Dangler, 1982; Roloff \& Denardin, 1994). O objetivo deste trabalho foi obter uma equação que, envolvendo variáveis de fácil determinação, estimasse o valor do fator $\mathrm{K}$ de solos com horizonte B textural (Bt) de diferentes regiões do Brasil.

\section{MATE RIAL E MÉTODOS}

Foram coletadas amostras não destorroadas dos horizontes A e Bt de solos com horizonte B textural de várias regiões geográficas do Brasil, cujos valores de erodibilidade (fator $K$ ) foram determinados diretamente no campo. A dassificação, a localização e o val or do fator $K$ observado estão relacionados no trabal ho de Marques et al. (1997).

$\mathrm{Na}$ fração TFSA (inferior a $2 \mathrm{~mm}$ ), foram feitas análises do complexo sortivo, $\mathrm{pH}$ (em água e em $\mathrm{KCl} 1 \mathrm{~mol} \mathrm{~L}^{-1}$ ) e carbono orgânico (Vettori, 1969; EMBRAPA, 1979). Nesta mesma fração, foram determinados os teores de $\mathrm{SiO}_{2}, \mathrm{Al}_{2} \mathrm{O}_{3}, \mathrm{Fe}_{2} \mathrm{O}_{3}, \mathrm{TiO}_{2} \mathrm{e}$ $\mathrm{P}_{2} \mathrm{O}_{5}$, extraídos pelo ataque sulfúrico (EMBRAPA, 1979). A atividade da argila foi cal culada subtraindose da CTC a pH 7 a contribuição da matéria orgânica ecorrigindo-se o resultado pel o teor de argila naTFSA.

A densidade de partículas foi determinada pelo método do balão vol umétrico (Blake \& Hartge, 1986). Pelo método de Grohmann \& Raij (1977), foram determinadas as seguintes frações granulométricas: acima de 5,66; 5,66 a 4,76; 4,76 a 2,83; e 2,83 a 2 mm. $\mathrm{Na}$ TFSA, foram feitas análises granulométricas e fracionamento de partículas, utilizando-se o método da pipeta (Day, 1965), com e sem o emprego de dispersantequímico ( $\mathrm{NaOH} 1 \mathrm{~mol} \mathrm{~L}^{-1}$ ). A fração areia foi dividida em: areia muito grossa (2 a $1 \mathrm{~mm}$ ); areia grossa (1 a 0,5 $\mathrm{mm})$; areia média $(0,5$ a $0,25 \mathrm{~mm})$, areia fina $(0,25$ a $0,105 \mathrm{~mm})$, areia muito fina $(0,105$ a $0,074 \mathrm{~mm})$, eareia muitíssi mo fina $(0,074$ a 0,053 mm).

Agregados de diâmetro entre 7,93 e 4,76 mm foram submetidos ao teste de estabilidade de agregados em água, através de peneiramento (Kemper \& Rosenau, 1986). A partir da quantidade de agregados retidos 
em cada peneira $(2 ; 1 ; 0,5 ; 0,25 ; \mathrm{e} 0,105 \mathrm{~mm})$, calcul ouse seu diâmetro médio geométrico (DMG) por meio da seguinte equação:

$$
D M G=\exp \left(\Sigma w_{i} \log x_{i}\right)\left(\Sigma w_{i}\right)^{-1}
$$

onde: $w_{i}=$ massa dos agregados de cada classe de tamanho (g); $x_{i}=$ diâmetro de cada classe detamanho (mm).

Empregaram-se três tipos de tratamentos da amostra de agregados para a análise de estabilidade: (1) sem pré-umedecimento; (2) com pré-umedecimento lento através de capilaridade, e (3) com préumedecimento lento através de capilaridade e descontando-se a contribuição da fração (dispersa em $\mathrm{NaOH} 1 \mathrm{~mol}^{-1}$ ) de tamanho superior ao da peneira em que os agregados estavam retidos (Angulo, 1983). Todas as análises anteriormente descritas foram realizadas em ambos os horizontes com quatro repetições.

Agregados do horizonte $A$ de 7,93 a 4,76 mm de diâmetro foram sujeitos ao teste de resistência ao impacto de gotas de chuva (Angulo, 1983). Esses agregados foram col ocados num suporte com abertura de malha de $4 \mathrm{~mm}$ sob um gotejador posicionado a $1,725 \mathrm{~m}$ de altura (Figura 1 ). Gotas de $4,17 \mathrm{~mm}$ de diâmetro atingiram os agregados com energia cinética igual a 0,296 mJ efreqüência de 1,65 $\pm 0,11$ gotas $\mathrm{s}^{-1}$. Os agregados foram considerados destruídos quando passaram pela abertura do suporte, enquanto os agregados que não passaram por essa abertura, após impacto de 300 gotas, foram considerados resistentes. O índice de estabilidade de agregados (IEA) foi calculado conformeAngulo (1983). O IEA foi expresso na forma original (Angulo, 1983), ignorando-se os agregados compostos de cascal ho de diâmetrosuperior a $4 \mathrm{~mm}$. Esta análise foi realizada com os agregados secos ao ar e em cinco repetições (dez agregados por repetição).

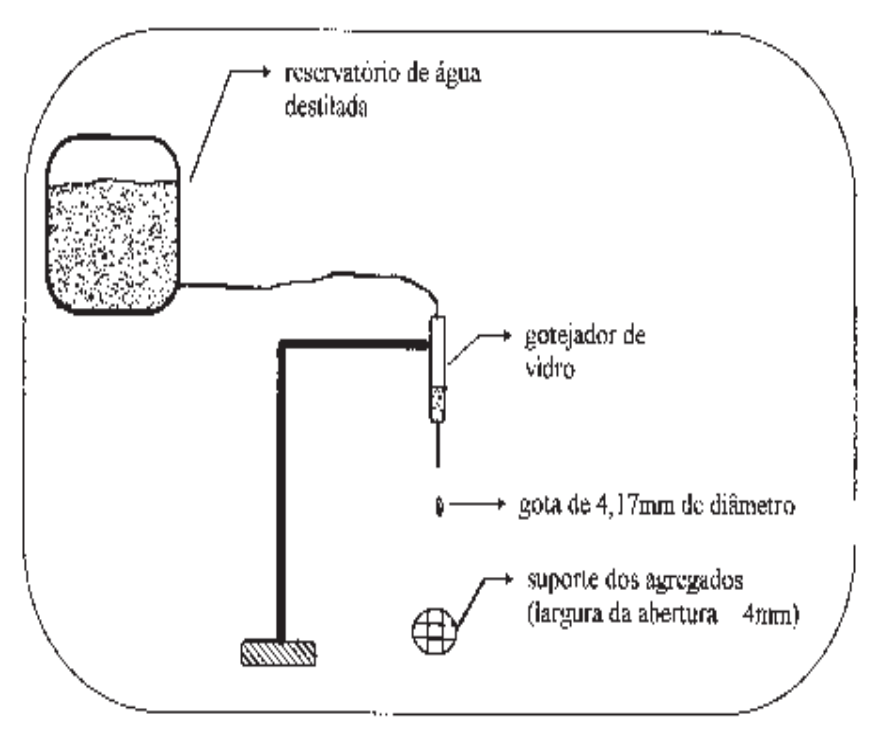

Figura 1. Esquema do gotejador utilizado no teste de resistência ao impacto de gotas (fora de escala).
Com as informações contidas na descrição morfológica dos perfis de cada solo, foram codificadas a estrutura do horizonteA ea permeabilidade do solo, segundo Wischmeier et al. (1971). A permeabilidade dos horizontes $\mathrm{A}$ e Bt foi estimada pelo método de Galindo \& Margolis (1989), obtendo-se também o produto da multiplicação da permeabilidade dos dois horizontes. Alternativamente, atribuiu-se às classes de drenagem - bem drenado, moderadamentedrenado eimperfeitamente drenado (únicas presentes nos solos estudados) - as notas 5, 4 e 3, respectivamente. Para quantificar a estrutura do solo, deram-se notas às diferentes classes de tamanho (muito grandea muito pequena), grau (forte a fraco) e tipo (granular, blocos etc.). A codificação do tamanho foi feita da maneira que se segue: grande $=8$; média $=6$; pequena $=4$; muito pequena $=2$; e maciça ou grãos simples $=1$. E mbora maciça e grãos simples sejam tipo de estrutura enão tamanho, normalmente, quando esses tipos são identificados, não se descreve o tamanho, razão por que foram codificados como 1 . Assim, os códigos da estrutura foram compostos pelas notas de cada uma dessas características (tamanho, grau etipo) ou combinações delas. $\mathrm{Na}$ combinação de dois atributos da estrutura (por exemplo, o tamanho: pequena e muito pequena), o valor médio das notas foi considerado.

No desenvolvimento de model os de estimativa do fator $\mathrm{K}$, consideraram-se como variáveis independentes: (1) os resultados das análises realizadas nas amostras dos horizontes dos diferentes solos; (2) os códigos de permeabilidade e estrutura; (3) as combinações de variáveis usadas com melhores resultados nos modelos propostos por Wischmeier et al. (1971), El-Swaify \& Dangler (1976), Denardin (1990) e Rol off \& Denardin (1994); e (4) combinações de variáveis criadas nestetrabal ho. I sso resultou num número total de variáveis independentes igual a 95. Os fatores K observados (Marques et al., 1997) foram considerados como variáveis dependentes. As equações receberam os valores de todas as variáveis e forneceram o valor do fator $\mathrm{K}$ em unidades do S.I. (t h (MJ mm)-1). U sou-se o programa estatísticoSAS, tendo sido os model os ajustados por meio de análise de regressão linear múltipla pelo processo de "stepwise". O nível mínimo de significância para inclusão ou exclusão de uma variável no modelo foi de 15\% (Denardin, 1990).

\section{RESULTADOS E DISCUSSÃO}

Das 95 variáveis testadas, 23 tomaram parte em, pelo menos, uma das 20 equações obtidas. A descrição dessas 23 variáveis encontra-se no quadro 1 , na mesma seqüência em quetomaram parte nos modelos. Conforme pode ser observado, o número de variáveis oriundas do horizonteA é, aproximadamente, o mesmo que do Bt. É possível, então, que um dos motivos capaz de explicar a falta de adequação dos métodos indiretos para a estimativa dofator $\mathrm{K}$ desol os com Bt (Marques, 1996), com exceção da estimativa da drenagem 
inferida com base no perfil do solo, seja o fato de os model os existentes não levarem em consideração as demais propriedades do horizonte subsuperficial. No entanto, isso não significa quesejam as características do horizonte Bt as responsáveis funcionais pela erodi bilidade. E mbora não exista, nem seja necessário existir, relação de causa e efeito entre as variáveis selecionadas e o fator K (Römkens, 1987), fica claro que a maioria delas tem participação nos processos que governam a erosão, não sendo, no seu conjunto, muito diferentes das selecionadas em outros estudos (Wischmeier \& Mannering, 1969; Wischmeier et al., 1971; El-Swaify \& Dangler, 1976; Römkens et al., 1977; Denardin, 1990).

Dos componentes caracterizadores da estrutura do solo (tamanho, grau e tipo) ou combinações deles, o tamanho apresentou mel hor correlação com o fator $K$ (Figura 2), sendo a primeira variável a ser incluída no modelo (Quadro 1). No horizonte A dos solos estudados, não se verificou a presença do tamanho muito grande. Nos trabalhos de Silva et al. (1994, 1995), solos com unidades estruturais menores, embora apresentassem maior quantidade de solo salpicada, mostravam menor erodibilidade, concordando com os resultados obtidos neste trabal ho. Possivelmente, as partículas sal picadas desses solos eram constituídas das próprias unidades estruturais, as quais, com o sal pico, apenas mudavam de posição sem alterar a estrutura da camada superficial, o que poderia levar à redução da infiltração. Por outro lado, quanto maior o tamanho dos agregados, mais fracas as forças que unem suas partículas (Oades, 1993). Assim, as partículas que formam os agregados grandes, quando estes são expostos ao impacto das gotas de chuva, são deslocadas mais facilmente e al ojam-se entre as unidades maiores, o que leva a um rápido selamento superficial e, conseqüentemente, à maior erosão (Le Bissonnais et al., 1989; Roth \& Eggert, 1994).

O tamanho das unidades estruturais do horizonte A pode ser facilmente determinado no campo (Lemos \& Santos, 1996) ou por meio das informações constantes na descrição morfológica dos perfis dos solos. Nestetrabalho, a estrutura foi analisada, graças a descrições morfológicas real izadas, em várias épocas, por pedól ogos das mais diversas institui ções e, mesmo

\section{Quadro 1. Descrição das variáveis incluídas nas equações}

\begin{tabular}{|c|c|}
\hline Variáveis & Descrição \\
\hline $\mathrm{X}_{1}$ & Código do tamanho da estrutura do horizonte A (adimensional) \\
\hline $\mathrm{X}_{2}$ & Silte disperso em água do horizonte $\mathrm{Bt}\left(\mathrm{g} \mathrm{kg}^{-1}\right)$ multiplicado por $\mathrm{X}_{1}$ \\
\hline $\mathrm{X}_{3}$ & $\begin{array}{l}\text { Partículas maiores que } 0,1 \mathrm{~mm} \text { dispersas em NaOH } 1 \text { mol L-1 }\left(\mathrm{g} \mathrm{kg}^{-1}\right) \text { multiplicadas por } \mathrm{C} \text { orgânico } \\
\left(\mathrm{g} \mathrm{kg}^{-1}\right) \text { do horizonte A (Denardin, 1990) }\end{array}$ \\
\hline $\mathrm{X}_{4}$ & C orgânico $\left(\mathrm{g} \mathrm{kg}^{-1}\right)$ multiplicado pela $A M G^{(1)}$ dispersa em água $\left(\mathrm{g} \mathrm{kg}^{-1}\right)$ do horizonte $A$ \\
\hline$X_{5}$ & $\begin{array}{l}\text { AMF (2) }\left(\mathrm{g} \mathrm{kg}^{-1}\right)+\mathrm{AMMF}^{(3)}\left(\mathrm{g} \mathrm{kg}^{-1}\right)+\text { silte }\left(\mathrm{g} \mathrm{kg}^{-1}\right) \text { multiplicados pela argila }\left(\mathrm{g} \mathrm{kg}^{-1}\right) \text { do horizonte } \mathrm{A} \\
\text { com dispersão em água }\end{array}$ \\
\hline $\mathrm{X}_{6}$ & DMG ${ }^{(4)}$ sem pré-tratamento do horizonte Bt (mm) \\
\hline $\mathrm{X}_{7}$ & Atividade da argila do horizonte $\mathrm{Bt}\left(\mathrm{mmol}_{\mathrm{c}} \mathrm{kg}^{-1}\right)$ \\
\hline $\mathrm{X}_{8}$ & AMG dispersa em água do horizonte $\mathrm{Bt}\left(\mathrm{g} \mathrm{kg}^{-1}\right)$ \\
\hline $\mathrm{X}_{9}$ & $\mathrm{Fe}_{2} \mathrm{O}_{3}$ extraído pelo ataque sulfúrico do horizonte $\mathrm{Bt}\left(\mathrm{g} \mathrm{kg}^{-1}\right)$ \\
\hline$X_{10}$ & C orgânico do horizonte Bt $\left(\mathrm{g} \mathrm{kg}^{-1}\right)$ \\
\hline$X_{11}$ & DMG com pré-tratamento, descontando-se o efeito da areia, do horizonte Bt (mm) \\
\hline $\mathrm{X}_{12}$ & $\mathrm{SiO}_{2}$ extraída pelo ataque sulfúrico do horizonte $\mathrm{Bt}\left(\mathrm{g} \mathrm{kg}^{-1}\right)$ \\
\hline $\mathrm{X}_{13}$ & Código da drenagem proposto (adimensional) \\
\hline $\mathrm{X}_{14}$ & Fração entre 5,66 e 4,76 mm dispersa em NaOH $1 \mathrm{~mol} \mathrm{L-1}$ do horizonte $\mathrm{A}\left(\mathrm{g} \mathrm{kg}^{-1}\right)$ \\
\hline $\mathrm{X}_{15}$ & DMG com pré-tratamento do horizonte Bt (mm) \\
\hline $\mathrm{X}_{16}$ & AG(5) dispersa em água do horizonte $\mathrm{Bt}\left(\mathrm{g} \mathrm{kg}^{-1}\right)$ \\
\hline $\mathrm{X}_{17}$ & Atividade da argila do horizonte $\mathrm{A}\left(\mathrm{mmol}_{\mathrm{c}} \mathrm{kg}^{-1}\right)$ \\
\hline $\mathrm{X}_{18}$ & Código da estrutura de Wischmeier et al. (1971) (adimensional) \\
\hline $\mathrm{X}_{19}$ & Código da permeabilidade de Galindo \& Margolis (1989) do horizonte A (adimensional) \\
\hline$X_{20}$ & $\mathrm{AF}{ }^{(6)}$ dispersa em água do horizonte $\mathrm{A}\left(\mathrm{g} \mathrm{kg}^{-1}\right)$ \\
\hline $\mathrm{X}_{21}$ & DMG com pré-tratamento do horizonte $A(\mathrm{~mm})$ \\
\hline$X_{22}$ & Índice de floculação do horizonte Bt $\left(\mathrm{g} \mathrm{g}^{-1}\right)$ \\
\hline $\mathrm{X}_{23}$ & Relação silte/argila dispersos em água do horizonte A ( $\left.\mathrm{g} \mathrm{g}^{-1}\right)$ \\
\hline
\end{tabular}

Frações de tamanho correspondentes a: ${ }^{(1)}$ areia muito grossa, ${ }^{(2)}$ areia muito fina; ${ }^{(3)}$ areia muitíssimo fina; ${ }^{(4)}$ Diâmetro médio geométrico;

(5) areia grossa; e $e^{(6)}$ areia fina. 
assim, forneceu resultados consistentes, oque mostra ser este um atributo confiável. A alta correlação encontrada entre otamanho das unidades estruturais e o fator $K$ ressalta a importância da descrição morfológica dos perfis de solo.

E mbora Bruce-Okine \& Lal (1975) tenham proposto a resistência dos agregados ao gotejamento como um bom índice da erodibilidade do solo, esse parâmetronão se mostrou bem rel acionado com ofator K, contrariando os resultados de Angulo (1983). Por outro lado, a presença do DMG, tanto do horizonte $A$ quanto do Bt, entre as variáveis selecionadas atesta a importância das variáveis estruturais no processo erosivo (Angulo, 1983), já que este é tido como um indicador da persistência das unidades estruturais no solo (Puget et al., 1995). I gwe et al. (1995) não encontraram correlação entre o fator $\mathrm{K}$ e a estabilidade estrutural nos solos tropicais por eles estudados, porém utilizaram para determinação do fator $\mathrm{K}$ o nomograma de Wischmeier et al. (1971). A permeabilidade do sol o codi ficada por Wischmeier et al. (1971) não foi incluída entre as variáveis selecionadas. Denardin (1990) inseriu esta variável em um de seus model os, contudo a baixa correl ação entre ela e o fator $K(r=0,26)$ talvez seja uma das causas da ineficiência do model o desse autor, quando aplicado a esses solos (Marques, 1996). Tanto o método de estimativa da permeabilidade de Galindo \& Margol is (1989) quanto o proposto neste trabal ho foram selecionados para o modelo (codificação das classes de drenagem: bem drenado $=5$; moderadamente drenado $=4$; e imperfeitamente drenado $=3$ ). Ambos são baseados na descrição morfológica dos perfis dos solos, o que reforça o discutido anteriormente, embora esses dois termos (drenagem e permeabilidade) não possam ser considerados equivalentes. A presença da fração 5,66 a 4,76 mm, entre as variáveis selecionadas, revela o controle da erosão exercido pela fração grosseira na superfície do solo. Estes reduzem a erosão aumentando a infiltração de água no solo, reduzindo a desagregação provocada pel o impacto das gotas de chuva, através da proteção (Chow \& Rees, 1995), além de apresentar maior massa para ser transportada.
Entre as combinações de variáveis criadas por outros autores (Wischmeier et al., 1971; El-Swaify \& Dangler, 1977; Denardin, 1990; Roloff \& Denardin, 1994), apenas a variável $X_{3}$, criada por Denardin (1990), foi selecionada para o modelo. A variável M ((silte + areia muito fina) $\times(1.000$ - argila)) apresentou uma correlação elevada com a erodi bilidade em outros trabal hos (Wischmeier et al., 1971; Römkens et al., 1977). Contudo, ao que parece, essa variável acha-se relacionada com a erodibilidade quando são incluídos na análise apenas solos de textura média (EI-Swaify \& Dangler, 1977; Denardin, 1990; Rol off \& Denardin, 1994).

No quadro 2, observam-se al gumas das equações obtidas, as quais são mostradas em virtude de possuírem el evados coeficientes de determinação, sem, contudo, terem um número excessivo de variáveis. Obviamente, à medida que novas variáveis são acrescidas ao modelo, o coeficiente de determinação aumenta, atingindo 1,00 quando 20 das 23 variáveis listadas no quadro 1 foram incluídas (equação não mostrada). As variáveis $X_{6}, X_{10}$ e $X_{9}$ foram removidas do modelo após a entrada da variável $X_{12}$. Os coeficientes de determinação obtidos são da mesma magnitude que os encontrados em outros trabal hos (Wischmeier et al., 1971; El-Swaify \& Dangler, 1977; Römkens et al., 1977; Denardin, 1990). A complexidade das equações éapenas apar ente, pois sua linearidade facilita muito os cál culos. Além disso, existe tendência de os model os de previsão de perdas de solo serem utilizados por meio de recursos computacionais (Roloff \& Clivati, 1995; Yoder \& Lown, 1995), o que reduz o trabalho de cálculo. O baixo número de variáveis foi apontado por Silva et al. (1994) como uma das causas da inadequação dos model os de Denardin (1990).

E mbora as equações geradas (Quadro 2) necessitem de número considerável de variáveis, estas são, sem exceção, de fácil determinação ou prontamente disponíveis nos boletins de levantamentos de solos. Possivelmente, apenas as variáveis $\mathrm{X}_{6}$ e $\mathrm{X}_{11}$ não sejam rotineiramentedeterminadas por todos os laboratórios de análise de solo nacionais. A escol ha da equação a ser empregada pode ser feita com base no coeficiente

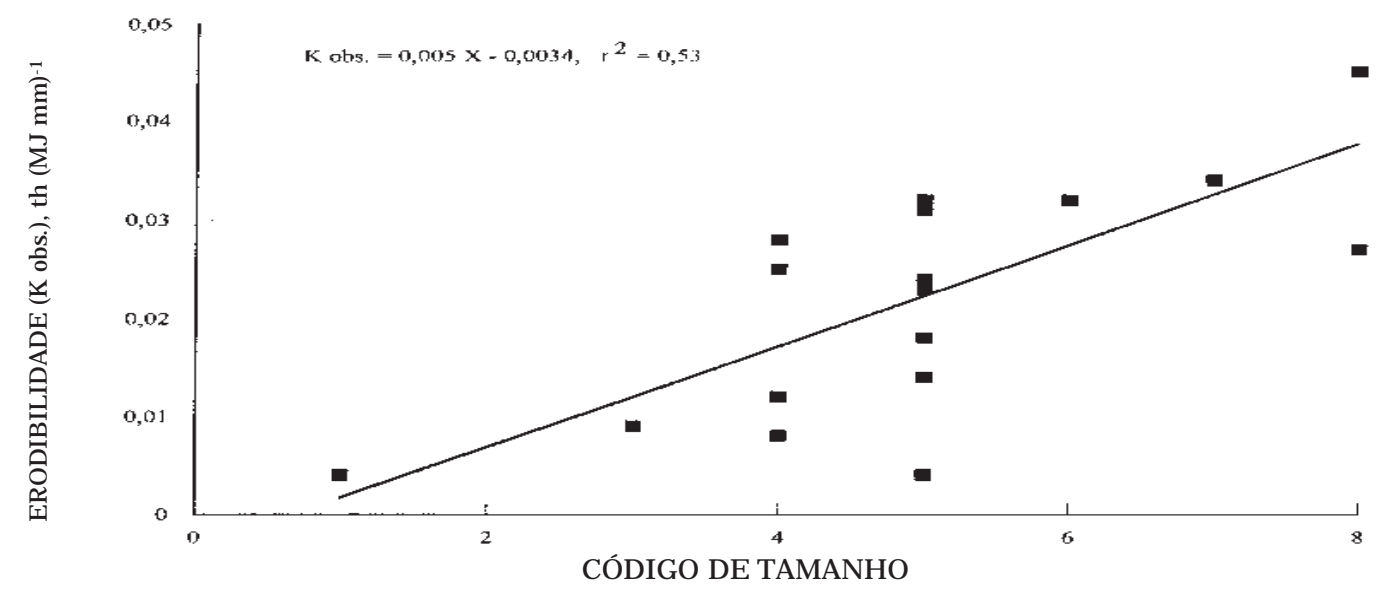

Figura 2. Relação entre o códi go do tamanho das unidades estruturais e o fator $\mathrm{K}$ observado (K obs.). 
de determinação (Quadro 2), em função da precisão desejada para o fator $\mathrm{K}$. O uso dessas equações restringe-sea solos geneticamentesemelhantes, cujos atributos não apresentem valores diversos dos abrangidos pelos solos aqui estudados.

Nos quadros 3 e 4, encontram-se os resultados das análises físicas, químicas e os códigos de estrutura e drenagem que compõem as variáveis presentes no quadro 2.
Nas figuras 3a e 3b, estão reunidos, respectivamente, os gráficos dos resíduos padronizados e a dispersão dos pontos (Kobs. x Kest.), usando-se a seguinte equação:

$$
\begin{aligned}
K= & -0,006+0,0074 X_{1}-0,855 \times 10^{-5} X_{2}+ \\
& +3,637 \times 10^{-6} X_{3}-1,878 \times 10^{-5} X_{4}+ \\
& +1,172 \times 10^{-7} X_{5}-0,012 X_{6}- \\
& -2,438 \times 10^{-4} X_{7}+1,107 \times 10^{-4} X_{8}
\end{aligned}
$$

\begin{tabular}{|c|c|c|c|c|c|c|c|c|c|c|c|c|}
\hline \multirow{3}{*}{ № } & \multirow{3}{*}{ Const. } & \multicolumn{10}{|c|}{ Variáveis } & \multirow[t]{3}{*}{$2^{2}$} \\
\hline & & \multirow[t]{2}{*}{$\mathrm{X}_{1}$} & $x_{2}$ & $X_{3}$ & $X_{4}$ & $X_{5}$ & $X_{6}$ & $X_{7}$ & $X_{8}$ & $X_{9}$ & $X_{10}$ & \\
\hline & & & $\times 10^{-5}$ & $\times 10^{-6}$ & $\times 10^{-5}$ & $\times 10^{-7}$ & & $\times 10^{-4}$ & $x 10^{-4}$ & $x 10^{-4}$ & $\times 10^{-4}$ & \\
\hline 1 & $-0,020$ & 0,0073 & $-0,684$ & 4,045 & $-1,465$ & 0,668 & & & & & & 0,79 \\
\hline 2 & $-0,016$ & 0,0080 & $-0,846$ & 3,736 & $-1,355$ & 0,989 & $-0,007$ & & & & & 0,85 \\
\hline 3 & $-0,007$ & 0,0078 & $-0,875$ & 3,281 & $-1,079$ & 0,928 & $-0,009$ & $-1,984$ & & & & 0,88 \\
\hline 4 & $-0,006$ & 0,0074 & $-0,855$ & 3,637 & $-1,878$ & 1,172 & $-0,012$ & $-2,438$ & 1,107 & & & 0,90 \\
\hline 5 & $-0,012$ & 0,0082 & $-1,044$ & 3,768 & $-2,122$ & 1,254 & $-0,015$ & $-2,819$ & 1,300 & 1,039 & & 0,94 \\
\hline \multirow[t]{3}{*}{6} & $-0,009$ & 0,0085 & $-1,148$ & 4,385 & $-2,446$ & 1,623 & $-0,017$ & $-3,104$ & 1,700 & 0,988 & $-8,659$ & 0,96 \\
\hline & & \multirow[t]{2}{*}{$X_{1}$} & $x_{2}$ & $X_{3}$ & $X_{4}$ & $X_{5}$ & $X_{11}$ & $x_{7}$ & $X_{8}$ & $X_{12}$ & $X_{13}$ & \\
\hline & & & $\times 10^{-5}$ & $\times 10^{-6}$ & $\times 10^{-5}$ & $\times 10^{-7}$ & & $x 10^{-4}$ & $\times 10^{-4}$ & $\times 10^{-4}$ & & \\
\hline 7 & 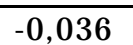 & 0,0081 & $-1,282$ & 7,096 & $-3,388$ & 2,078 & -0 , & $-5,333$ & 1,441 & 1,639 & & 0,97 \\
\hline 8 & $-0,029$ & 0,0081 & $-1,373$ & 7,621 & $-3,638$ & 2,221 & $-0,014$ & $-6,162$ & 1,551 & 1,935 & $-0,002$ & 0,98 \\
\hline
\end{tabular}

\begin{tabular}{|c|c|c|c|c|c|c|c|c|c|c|c|}
\hline \multirow{3}{*}{ Solo } & \multicolumn{9}{|c|}{ Horizonte A } & \multicolumn{2}{|c|}{ Horizonte B } \\
\hline & \multicolumn{4}{|c|}{ NaOH 1 mol L-1 } & \multicolumn{7}{|c|}{ Água } \\
\hline & $A M G^{(1)}$ & $\mathbf{A} \mathbf{G}^{(2)}$ & $A M^{(3)}$ & AF (4) & AMG & $A M F^{(5)}$ & $A M M F^{(6)}$ & Silte & Argila & AMG & Silte \\
\hline $\mathrm{N} \cong$ & & & & & & $-\mathrm{g} \mathrm{kg}^{-1}$ & 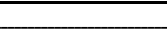 & & & & - \\
\hline $\begin{array}{r}1 \\
2 \\
3 \\
4 \\
5 \\
6 \\
7 \\
8 \\
9 \\
10 \\
11 \\
12 \\
13 \\
14 \\
15 \\
16 \\
17 \\
18 \\
19 \\
20 \\
21 \\
22\end{array}$ & $\begin{array}{r}43 \\
40 \\
4 \\
127 \\
20 \\
57 \\
18 \\
65 \\
16 \\
66 \\
57 \\
72 \\
53 \\
76 \\
23 \\
46 \\
14 \\
22 \\
5 \\
0 \\
52 \\
43\end{array}$ & $\begin{array}{r}134 \\
106 \\
45 \\
157 \\
132 \\
189 \\
50 \\
140 \\
97 \\
143 \\
137 \\
113 \\
120 \\
213 \\
45 \\
171 \\
47 \\
21 \\
87 \\
6 \\
155 \\
171\end{array}$ & $\begin{array}{r}192 \\
208 \\
134 \\
147 \\
138 \\
188 \\
116 \\
119 \\
180 \\
149 \\
135 \\
140 \\
128 \\
139 \\
60 \\
179 \\
69 \\
19 \\
230 \\
147 \\
123 \\
169\end{array}$ & $\begin{array}{r}328 \\
388 \\
199 \\
206 \\
138 \\
194 \\
265 \\
209 \\
316 \\
233 \\
199 \\
217 \\
183 \\
149 \\
278 \\
192 \\
89 \\
33 \\
279 \\
531 \\
147 \\
178\end{array}$ & $\begin{array}{r}31 \\
41 \\
5 \\
104 \\
12 \\
67 \\
19 \\
49 \\
13 \\
88 \\
67 \\
63 \\
33 \\
95 \\
29 \\
39 \\
31 \\
16 \\
8 \\
1 \\
63 \\
40\end{array}$ & $\begin{array}{r}94 \\
44 \\
41 \\
51 \\
17 \\
32 \\
107 \\
78 \\
94 \\
53 \\
52 \\
63 \\
51 \\
32 \\
167 \\
45 \\
27 \\
9 \\
56 \\
77 \\
39 \\
39\end{array}$ & $\begin{array}{r}26 \\
31 \\
67 \\
29 \\
12 \\
26 \\
90 \\
80 \\
81 \\
42 \\
42 \\
53 \\
44 \\
22 \\
125 \\
30 \\
26 \\
13 \\
39 \\
55 \\
18 \\
25\end{array}$ & $\begin{array}{r}103 \\
110 \\
451 \\
181 \\
382 \\
182 \\
233 \\
246 \\
177 \\
169 \\
208 \\
205 \\
215 \\
194 \\
174 \\
196 \\
408 \\
486 \\
181 \\
87 \\
244 \\
192\end{array}$ & $\begin{array}{r}70 \\
74 \\
68 \\
36 \\
331 \\
150 \\
109 \\
61 \\
51 \\
101 \\
151 \\
138 \\
190 \\
137 \\
90 \\
130 \\
238 \\
396 \\
64 \\
58 \\
168 \\
150\end{array}$ & $\begin{array}{r}26 \\
19 \\
24 \\
156 \\
6 \\
26 \\
61 \\
53 \\
22 \\
66 \\
71 \\
38 \\
22 \\
135 \\
25 \\
59 \\
12 \\
32 \\
45 \\
1 \\
95 \\
33\end{array}$ & $\begin{array}{l}426 \\
210 \\
419 \\
241 \\
759 \\
174 \\
242 \\
196 \\
191 \\
262 \\
251 \\
266 \\
213 \\
204 \\
249 \\
184 \\
825 \\
335 \\
234 \\
193 \\
243 \\
544\end{array}$ \\
\hline
\end{tabular}

Quadro 2. Variáveis e coeficientes de determi nação $\left(r^{2}\right)$ das equações obti das por "stepwise"

Quadro 3. Atributos granulométricos dos solos estudados

Frações de tamanho correspondentes a: $:^{(1)}$ Areia muito grossa $(1$ a $2 \mathrm{~mm}) ;{ }^{(2)}$ Areia grossa $(0,5$ a $1 \mathrm{~mm}) ;{ }^{(3)}$ Areia média $(0,25$ a $0,50 \mathrm{~mm}) ;$

${ }^{(4)}$ Areia fina $(0,1$ a $0,25 \mathrm{~mm}) ;{ }^{(5)}$ Areia muito fina $(0,05$ a $0,1 \mathrm{~mm}) ;{ }^{; 6)}$ Areia muitíssimo fina $(0,074$ a $0,053 \mathrm{~mm})$. 
Quadro 4. Teores de C orgânico, $\mathrm{Fe}_{2} \mathrm{O}_{3}$ e $\mathrm{SiO}_{2}$ extraídos pelo ataque sulfuríco do horizonte $\mathrm{Bt}$, diâmetro médio geométrico (DMG) dos agregados do horizonte Bt, atividade da argila do horizonte Bt (T arg.), códigos da estrutura do horizonte A (E) e da drenagem do solo (D)

\begin{tabular}{|c|c|c|c|c|c|c|c|c|c|}
\hline \multirow{2}{*}{ Solo } & \multicolumn{2}{|c|}{ C orgânico } & \multirow{2}{*}{$\begin{array}{c}\mathbf{T} \\
\text { argila }\end{array}$} & \multirow{2}{*}{$\mathrm{Fe}_{2} \mathrm{O}_{3}$} & \multirow{2}{*}{$\mathrm{SiO}_{2}$} & \multirow{2}{*}{$E^{(1)}$} & \multirow{2}{*}{$D^{(2)}$} & \multicolumn{2}{|c|}{ DMG } \\
\hline & Hor. A & Hor. Bt & & & & & & $s / p^{(3)}$ & $c / p s / a^{(4)}$ \\
\hline 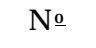 & \multicolumn{2}{|c|}{$-\mathrm{g} \mathrm{kg}^{-1}-$} & $\mathrm{mmol}_{\mathrm{c}} \mathrm{kg}^{-1}$ & \multicolumn{2}{|c|}{$-\mathrm{g} \mathrm{kg}^{-1}-$} & & & \multicolumn{2}{|c|}{$\longrightarrow \mathrm{mm}=$} \\
\hline $\begin{array}{r}1 \\
2 \\
3 \\
4 \\
5 \\
6 \\
7 \\
8 \\
9 \\
10 \\
11 \\
12 \\
13 \\
14 \\
15 \\
16 \\
17 \\
18 \\
19 \\
20 \\
21 \\
22\end{array}$ & $\begin{array}{r}5 \\
4 \\
13 \\
15 \\
14 \\
14 \\
7 \\
13 \\
4 \\
9 \\
9 \\
9 \\
11 \\
17 \\
7 \\
2 \\
28 \\
21 \\
9 \\
6 \\
13 \\
12\end{array}$ & $\begin{array}{r}4 \\
4 \\
6 \\
4 \\
6 \\
5 \\
3 \\
4 \\
6 \\
4 \\
4 \\
5 \\
7 \\
13 \\
4 \\
3 \\
3 \\
9 \\
7 \\
4 \\
11 \\
3\end{array}$ & $\begin{array}{r}147 \\
87 \\
337 \\
213 \\
22 \\
109 \\
119 \\
343 \\
240 \\
555 \\
213 \\
161 \\
84 \\
240 \\
377 \\
137 \\
269 \\
75 \\
322 \\
165 \\
118 \\
64\end{array}$ & $\begin{array}{r}30 \\
20 \\
62 \\
61 \\
101 \\
55 \\
66 \\
52 \\
44 \\
71 \\
68 \\
43 \\
66 \\
24 \\
76 \\
56 \\
97 \\
58 \\
46 \\
38 \\
85 \\
88\end{array}$ & $\begin{array}{l}153 \\
160 \\
186 \\
231 \\
248 \\
120 \\
185 \\
148 \\
128 \\
178 \\
154 \\
181 \\
209 \\
134 \\
195 \\
225 \\
231 \\
271 \\
163 \\
130 \\
192 \\
226\end{array}$ & $\begin{array}{l}4 \\
8 \\
5 \\
7 \\
8 \\
5 \\
5 \\
5 \\
4 \\
4 \\
1 \\
4 \\
4 \\
5 \\
3 \\
5 \\
5 \\
6 \\
5 \\
1 \\
5 \\
4\end{array}$ & $\begin{array}{l}5 \\
5 \\
5 \\
5 \\
4 \\
4 \\
4 \\
3 \\
3 \\
5 \\
5 \\
5 \\
5 \\
4 \\
4 \\
4 \\
5 \\
5 \\
5 \\
5 \\
5 \\
4\end{array}$ & $\begin{array}{l}0,40 \\
0,32 \\
0,45 \\
1,37 \\
0,62 \\
0,60 \\
1,83 \\
0,71 \\
0,94 \\
0,51 \\
0,85 \\
0,89 \\
1,85 \\
0,58 \\
0,45 \\
1,53 \\
0,34 \\
1,64 \\
0,37 \\
0,48 \\
1,11 \\
0,57\end{array}$ & $\begin{array}{l}0,65 \\
0,35 \\
0,85 \\
2,45 \\
2,47 \\
1,39 \\
2,6 \\
0,66 \\
0,65 \\
0,24 \\
1,09 \\
1,01 \\
3,78 \\
0,87 \\
0,38 \\
2,46 \\
0,60 \\
4,30 \\
0,91 \\
0,63 \\
2,29 \\
1,79\end{array}$ \\
\hline
\end{tabular}

(1) Valores segundo as características estruturais; ${ }^{(2)}$ Valores segundo a classe de drenagem: 5 = bem drenado, $4=$ moderadamente drenado, 3 =imperfeitamente drenado; ${ }^{(3)}$ sem pré-umedecimento; ${ }^{(4)}$ com pré-umedecimento lento e descontando-se a contribuição da fração de tamanho superior ao da peneira em questão.
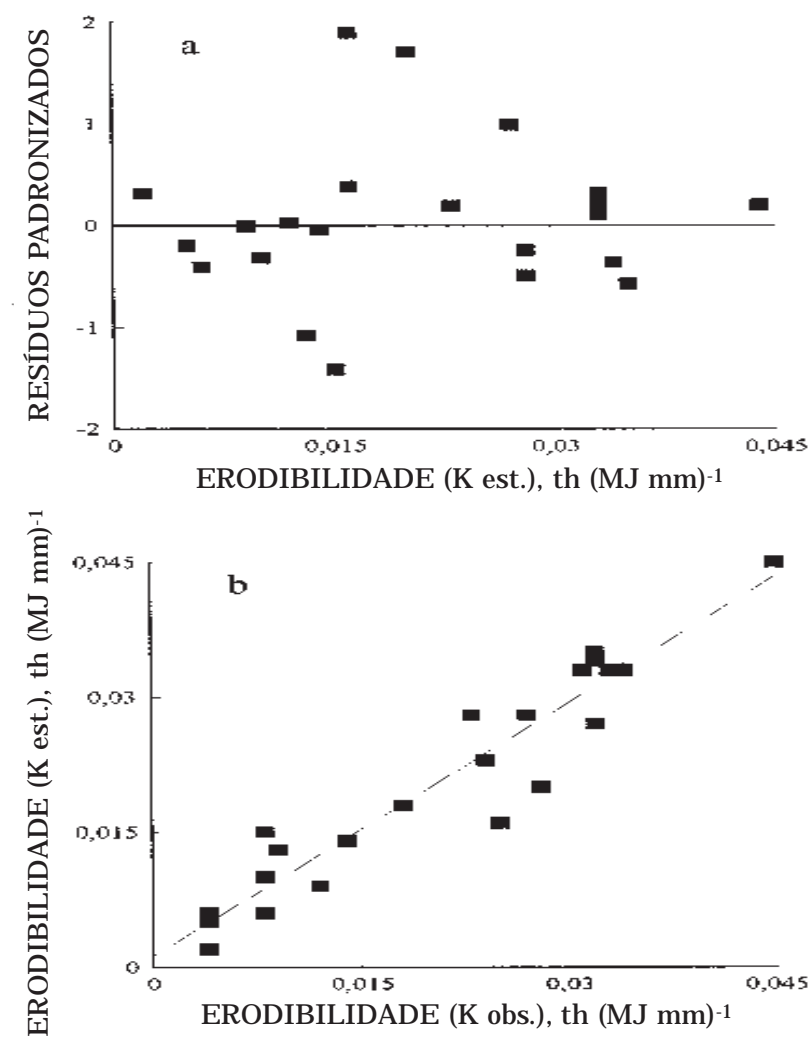

Figura 3. Resíduos padronizados (a) e relação entre os valores observados e estimados do fator $K$ pela equação 4 (b). Na figura 3 b o traço contínuo representa a linha 1:1.
As variáveis relacionadas no model o foram descritas no quadro 1. Esta equação foi escolhida por ser, dentre as de mais alto ${ }^{2}$, a que possui variáveis mais facilmente adaptáveis à rotina. Na figura 3a, observase que a distribuição dos resíduos padronizados está dentro da faixa 2 e-2, indicando serem el es aceitáveis (Neter \& Wassermann, 1974). Como os erros estão uniformemente distribuídos ao longo dos valores de $K$ estimados, pode-se afirmar que não existetendência de super ou subestimação dos val ores da erodibilidade para certos valores estimados. Embora a maioria dos valores de erodibilidade estimados esteja bastante próxima dos observados $\left(r^{2}=0,90\right)$, para al guns sol os (1, 12 e 22), esta equação ainda mostra certa inadequação (Figura 3b). Na figura 3b, optou-se por não mostrar o intervalo de confiança do val or predito, visto que, para cada solo, ele assume diferentes amplitudes conforme os val ores de $X_{1}$ a $X_{8}$ distanciamse da média dos 22 solos. Entre todos os valores observados, apenas os sol os 1, 12 e 22 achavam-se além do intervalo de confiança do fator $\mathrm{K}$ estimado.

\section{CONCLUSÕES}

1. A partir das variáveis selecionadas, tanto do horizonteA quanto do Bt, eda distribuição do tamanho das partículas, obtida com esem dispersante químico, foi possível estimar satisfatoriamentea erodibilidade dos solos brasileiros com horizonte B textural. 
2. As informações contidas na descrição morfológica dos perfis de solo, especialmente as referentes à estrutura, bem como os teores de carbono orgânico, foram de grande importância para a determinação indireta da erodibilidade de solos brasileiros com horizonte B textural.

\section{AGRADECIMENTOS}

Os autores agradecem aos pesquisadores e professores D. J. Reinert (UFSM), E. Margolis (UFRPE), G.R. Curcio (EMBRAPA), I.B. Chaves el.F. Silva (UFPB), J.R.C. Silva (UFC), L.H.C. Anjos (UFRRJ ), M. Resende (UFV), N. Kämpf (UFRGS), R.C. Alvarenga (EMBRAPA) e S.C.F. Dechen (IAC) que, gentilmente, enviaram amostras de solo.

\section{LITE RATURA CITADA}

ANGULO, J.R. Relações entre a erodibilidade e algumas propriedades de sol os brasileiros. Curitiba, UFPR, 1983. 154p. (Dissertação de Mestrado)

BLAKE, G.R. \& HARTGE, K.H. Particle density. In: KLUTE, A., ed. Methods of soil analysis. Madison, American Society of Agronomy, 1986. v.1, p.377-382.

BRUCE-OKINE, E. \& LAL, R. Soil erodibility as determined by raindrop technique. Soil Sci., Baltimore, 119:149-157, 1975.

CHOW, T.L. \& REES, H.W. Effects of coarse-fragment content and size on soil erosion under simulated rainfall. Can.J. Soil Sci., Ottawa, 75:227-232, 1995.

DAY, P.R. Particle fractionation and particle - size analysis. In: BLACK, C.A., ed. Methods of soil analysis. Madison, American Society of Agronomy, 1965. v.1, p.545-566.

DENARDIN, J.E. Erodibilidade de solo estimada por meio de parâmetros físicos equímicos. Piracicaba, ESALQ, 1990. 81p. (Tese de Doutorado)

EL-SWAIFY, S.A. \& DANGLER, E.W. Erodibilities of selected tropical soils in relation to structural and hydrologic parameters. In: NATIONAL CONFERENCE ON SOIL EROSION, 1976, West Lafayette. Proceedings. Ankeny, SWCS, 1977. p.105-104.

EL-SWAIFY, S.A. \& DANGLER, E.W. Rainfall erosion in the tropics: a state of art. In: KREBS, D.M., ed. Determinants of soil Ioss tolerance. Madison, American Society of Agronomy, 1982. p.1-25.

EMPRESA BRASILEIRA DE PESQUISA AGROPECUÁRIA. Manual de métodos de análise de solos. Rio de J aneiro, EMBRAPA-SNLCS, 1979. n.p.

FOSTER, G.R.; MOLDENHAUER, W.C. \& WISCHMEIER, W.H. Transferability of U.S. technology for prediction and control of erosion in the tropics. In: American Society of Agronomy. Soil erosion and conservation in the tropics. Madison, ASA/ SSSA, 1982. p.135-149. (Special Publication, 43)

GALINDO, I.C.L. \& MARGOLIS, E. Tolerância de perdas por erosão para sol os do Estado de Pernambuco. R. bras. Ci. Solo, Campinas, 13:95-100, 1989.

GROHMANN, F. \& RAIJ , B. Dispersão e pré-tratamento para análise granulométrica de latossolos argilosos. R. bras. Ci. Solo, Campinas, 1:52-53, 1977.
HENKLAIN, J.C. \& FREIRE, O. Avaliação do método nomográfico para determinação da erodibilidade de latossolos do Estado do Paraná. R. bras. Ci. Solo, Campinas, 7:191-195, 1983.

IGWE, C.A.; AKAMIGBO, F.O.R. \& MBAGWU, J.S.C. Physical properties of soils of southeastern Nigeria and the role of someaggregating agents in their stability. Soil Sci., Baltimore, 160:431-441, 1995.

KEMPER, W.D. \& ROSENAU, R.C. Aggregate stability and size distribution. In: KLUTE, A., ed. Methods of soil analysis. Madison, American Society of Agronomy, 1986. v.1, p.425-442.

LE BISSONNAIS, Y.; BRUAND, A. \& J AMAGNE, M. Laboratory experimental study of soil crusting: relation between aggregate breakdown mechanisms and crust structure. Catena, Cremlingen, 16:377-392, 1989.

LEMOS, R.C. \& SANTOS, R.D. Manual de descrição e coleta de solo no campo. 3.ed. Campinas, SBCS, 1996. 83p.

LIMA, J.M.; CURI, N.; RESENDE, M. \& SANTANA, D.P. Dispersão do material de solo em água para avaliação indireta da erodibilidade em latossolos. R. bras. Ci. Solo, Campinas, 14:85-90, 1990.

LIMA, P.M.P. Í ndices de erodibilidade diretos e indiretos para dois latossol os do município de Lavras - Minas Gerais. Ci. Prát., Lavras, 15:186-193, 1991.

MARQUES, J.J .G.S.M.; CURI, N.; FERREIRA, M.M.; LIMA, J .M.; SILVA, M.L.N. \& CAROLINO de SÁ, M.A. Adequação de métodos indiretos para estimativa da erodibilidade de solos com horizonte B textural no Brasil. R. bras. Ci. Solo, Viçosa, 21:447-456, 1997.

MARQUES, J.J.G.S.M. Estimativas e comparações dos fatores erosividade das chuvas eerodibilidade de sol os com horizonte B textural no Brasil. Lavras, UF LA, 1996. 119p. (Dissertação de Mestrado)

NETER, . \& WASSE RMANN, W. Applied linear statistical models: regression, analysis of variance and experimental designs. Homewood, Richard D. Irwin, 1974. 842p.

OADES, J.M. The role of biology in the formation, stabilization and degradation of soil structure. Geoderma, Amsterdam, 56:377-400, 1993.

OBI, M.E.; SALAKO, F.K.\& LAL, R. Relative susceptibility of some southeastern Nigeria soils to erosion. Catena, Cremlingen, 16:215-225, 1989.

PUGET,P.; CHENU, C. \& BALESDENT,J .Total and young organic matter distributions in aggregates of silty cultivated soils. Eur. J. Soil Sci., Oxford, 46:449-459, 1995.

RENARD, K.G.; FOSTER, G.R.; YODER, D.C. \& McCOOL, D.K. RUSLE revised: status, questions, answers and the future. J. Soil Water Cons., Ankeny, 49:213-220, 1994.

ROLOFF, G. \& CLIVATI, A.A. Modelo de erosão e sedimentação para oParaná. In: CONGRESSO BRASILEIRO DE CIÊNCIA DO SOLO, 25., Viçosa, 1995. Resumos. Viçosa, SBCS/UFV, 1995. v.3, p.1782-1784.

ROLOFF, G. \& DENARDIN, J.E. Estimativa simplificada da erodibilidade do solo. In: REUNIÃO BRASILEIRA DE MANEJ O E CONSERVAÇÃO DO SOLO E DA ÁGUA, 10., Florianópolis, 1994. Resumos. Florianópolis, SBCS, 1994. p.146-147.

RÖMKENS, M.J.M. The soil erodibility factor: a perspective. In: HARLIN, J.M. \& BERARDI, G.M., eds. Agricultural soil loss: processes, policies and prospects. Boulder, Westview, 1987. p.125-149. 
RÖMKENS, M.J.M.; ROTH, C.B. \& NELSON, D.W. Erodibility of selected clay subsoils in relation to physical and chemical properties. Soil Sci. Soc. Am. J., Madison, 41:954-960, 1977.

ROTH, C.H. \& EGGERT, T. Mechanisms of aggregate breakdown involved in surface sealing, runoff generation and sediment concentration on loess soil Is. Soil Til. Res., Amsterdam, 32:253268, 1994.

SILVA, I.F.; ANDRADE, A.P. \& CAMPOS FILHO, O.R Erodibilidade de seis solos do semi-árido paraibano obtida com chuva simulada e método nomográfico. R. bras. Ci. Solo. Campinas, 10:283-287, 1986.

SILVA, M.L.N.; CURI, N.; MARQUES, J.J .G.S.M.; LIMA, L.A.; FERREIRA, M.M. \& LIMA, J.M. Resistência ao salpico provocado por impacto de gotas de chuva simulada em latossolos e sua relação com características químicas e mineralógicas. Ci. Prát., Lavras, 19:176-182, 1995.

SILVA, M.L.N.; CURI, N.; OLIVEIRA, M.S.; FERREIRA, M.M. \& LOMBARDI NETO, F. Comparação de métodos direto e indiretos para determinação da erodibilidade em latossolos sob cerrado. Pesq. Agropec. bras., Brasília, 29:1751-1761, 1994.
VETTORI, L. Métodos de análise de solos. Rio de J aneiro, Ministério da Agricultura-Equipe de Pedologia e Fertilidade do Solo, 1969. 24p. (Boletim Técnico, 7)

YODER, D. \& LOWN, J. The future of RUSLE: inside the new Revised Universal Soil Loss Equation. J. Soil Water Cons., Ankeny, 50:484-489, 1995.

YOUNG, R.A. \& MUTCHLER, C.K. Erodibility of some Minnesota soils. J. Soil Water Cons., Ankeny, 32:180-182, 1977.

WISCHMEIER, W.H. Use and misuse of the universal soil loss equation. J. Soil Water Cons., Ankeny, 31:5-9, 1976.

WISCHMEIER, W.H.; J OHNSON, C.B. \& CROSS, B.V. A soil erodibility nomograph for farmland and construction sites. J. Soil Water Cons., Ankeny, 26:189-193, 1971.

WISCHMEIER, W.H. \& MANNERING, J.V. Relation of soil properties to its erodibility. Soil Sci. Soc. Am. Proc., Madison, 33:131-137, 1969.

WISCHMEIER, W.H. \& SMITH, D.D. Predicting rainfall erosion Iosses: a guide to conservation planning, Washington, USDA, 1978. 58p. (Agriculture Handbook, 537) 